\title{
Zwanzig Jahre Armutsberichterstattung in Deutschland
}

\author{
Eine Fachtagung des Paritätischen Wohlfahrtsverbandes \\ blickte zurück und nach vorn
}

Ulrike Bauer

Ulrike Bauer ist Redakteurin der

Verbandszeitschrift »Der Paritätische« des Gesamtverbandes des Paritätischen Wohlfahrtsverbandes.

E-Mail nachrichten@paritaet.org
Zwanzig Jahre nachdem der Paritätische Wohlfahrtsverband seinen ersten Armutsbericht für die Bundesrepublik Deutschland vorgelegt hat, zog der Verband in einer Fachtagung eine ernüchternde Bilanz. Zwar werde es inzwischen weitgehend nicht mehr bestritten, dass es in Deutschland Armut gebe; das Ziel, diese wirkungsvoll zu bekämpfen, sei aber immer noch nicht erreicht.

Der Paritätische Wohlfahrtsverband stellte am 9. November 1989 seinen ersten Armutsbericht vor. Der Report erschien als Themenheft der Blätter der Wohlfahrtspflege 11-12/1989 unter dem Titel »... wessen wir uns schämen müssen in einem reichen Land ...«.

Damit gelang es dem Verband erstmals, Begriff und Ausmaß der Armut in Deutschland in die Öffentlichkeit zu transportieren, betonte Josef Schädle, heute stellvertretender Vorsitzender des Paritätischen Gesamtverbandes und damals Mitautor des Berichts zur Eröffnung der Fachtagung zu Stand und Perspektiven der Armutsberichterstattung. Sogar in die "Tagesschau « der ARD, in der Schabowskis historische Pressekonferenz zum Fall der Mauer gesendet wurde, schaffte es der Verband mit seinem Bericht.

»Ein Tabu war gebrochen «, so Schädle. Armut als Massenphänomen konnte nicht länger geleugnet werden. Nicht nur in der Politik, auch in der Bevölkerung hatten bis dahin viele ihre Augen vor der Tatsache verschlossen, dass angesichts von damals mehr als zwei Millionen Arbeitslosen und 600.000 Langzeitarbeitslosen Armut in Westdeutschland keine Randerscheinung war, die nur »Versager « traf. Die Bundesregierung verweigerte sich einer nationalen Armutsberichterstattung jedoch mit dem Argument, Armut könne nicht allgemeingültig definiert werden, und entzog sich so der Pflicht zu politischem Handeln. »Und das fehlende Wissen um die alläglichen Belastungen am Randes unseres Gemeinwesens machte es wiederum so leicht, Armut zu leugnen «, erinnerte der Hauptgeschäftsführer des Paritätischen, Dr. Ulrich Schneider, an die damalige Argumentation.

Zwölf Jahre dauerte es, bis die Bundesregierung 2001 einen Nationalen Armuts- und Reichtumsbericht vorlegte. Doch bei näherem Hinsehen, so Schneider, "will es scheinen, als seien uns unsere politischen Erfolge zum Teil zwischen den Fingern zerronnen ... Man kann nach 20 Jahren den Eindruck gewinnen, als stünden wir wieder am Anfang «. Zwar sei heute von Einkommensarmut, Ressourcenarmut, Teilhabearmut und Bildungsarmut die Rede, doch diese Zerfaserung des Armutsbegriffs habe ihm seine handlungsleitende Wirkung genommen.

\section{»Das Gute wird mit Verweis auf das vermeintlich Bessere unterlassen «}

»Die Ausdifferenzierung des Armutsbergriffs wird nicht selten politisch als Legitimation benutzt, um das Gute mit dem Verweis auf das möglicherweise Bessere zu unterlassen «, kritisierte der Hauptgeschäftsführer und Mitautor des ersten Armutsberichts des Paritätischen. "Jüngstes Beispiel ist das unselige Ausspielen von monetären Transferleistungen für arme Familien gegenüber notwendigen Infrastrukturleistungen ", so Schneider. »Die Devisen lauten: Geld allein macht nicht glücklich. Und: Bildung tut not. Dass der Schulbesuch jedoch keinen Wintermantel ersetzen kann, sollte eine selbstverständliche Einsicht sein, doch es wird zum Teil hartnäckig ignoriert. «

Schneider kritisierte, dass der Armutsund Reichtumsbericht der Bundesregierung nicht von einer unabhängigen Sachverständigen-Kommission erarbeitet wer- 
de. Die Erstellung durch das Arbeits- und Sozialministerium mache Rollenkonflikte unauflösbar. Der zuletzt vorgelegte 3 . Armutsbericht mache deutlich: »Das Bedürfnis, regierungsamtliches Handeln zu legitimieren, statt über Armut aufzuklären, hat offensichtlich obsiegt. « Die Vorlage des 3. Armutsberichts habe armutspolitisch mehr Schaden angerichtet als Vernünftiges bewirkt, kritisierte Schneider. »Die vorgelegten Gutachten und Daten wurden mit Interpretationen verse-

\section{"Lebensstandardansatz praktisch umsetzen «}

Armutsforscher Prof. Dr. Richard Hauser, der 1981 mit einer Arbeitsgruppe im Auftrag der damaligen Europäischen Gemeinschaft einen ersten Nationalen Armutsbericht für Deutschland erstellte, hält eine Weiterentwicklung der Armutsberichterstattung für erforderlich. Er bezeichnete die Veröffentlichung des ersten »Regionalen Armutsatlasses «, mit dem

\section{»Der erste Armutsbericht des Paritätischen hat ein Tabu gebrochen"}

hen, die sachlich und fachlich zum Teil kaum haltbar waren, jedoch die Bundesregierung ausschließlich in ein gutes Licht stellen sollten. «

\section{»Armutspolitisch mehr Schaden angerichtet als Vernünftiges bewirkt «}

Schneider warnte: Sollte die Bundesregierung künftig nicht bereit sein, den Bericht durch eine unabhängige Kommission erstellen zu lassen, müsse ernsthaft überlegt werden, »ob sich die Zivilgesellschaft ihre Armutsberichterstattung nicht wieder zurückholt«. Armutsberichte müssten Teil der Armutsbekämpfung sein; zugleich müssten sie die Menschen nicht nur erreichen, sondern sensibilisieren und motivieren, um gesellschaftliches und politisches Handeln auszulösen. »Wir brauchen eine Armutsberichterstattung, die die Menschen berührt. «

Reden und Audiomitschnitte der Tagung sind zu finden auf der Internetpräsenz des Paritätischen Gesamtverbandes http://www.paritaet.org unter » Tagungen \& Kongresse « im Bereich »Dokumentationen «. der Paritätische im Sommer 2009 die regionalen Ausmaße der Armut deutlich gemacht hat, als Pioniertat. Dagegen kritisierte auch er den 3. Armutsbericht der Bundesregierung. Im Vergleich $\mathrm{zu}$ den Gutachten, auf denen er basiere, sei der Bericht verniedlichend.

Hauser sagte, zu den künftigen Aufgaben einer fundierten Armutsberichterstattung müsse es gehören, die Ursachen materieller Armut tiefer zu analysieren und die Situation von Menschen in besonderen Lebenslagen - wie etwa Menschen mit Behinderung oder chronischen Erkrankungen oder Personen die in Alten- und Pflegeheimen leben - stärker zu berücksichtigen.

Darüber hinaus sei eine präzisere Erfolgskontrolle der steuer-, arbeitsmarktund sozialpolitischen Maßnahmen der Armutsbekämpfung erforderlich. Zur Festlegung der Armutsgrenze schlug er vor, auf den Lebensstandardansatz zurückzugreifen. Dabei werden in einer breit angelegten Umfrage zufällig ausgewählte Bürgerinnen und Bürger befragt, welche Güter und Leistungen sie für ein annehmbares Leben für erforderlich halten und ob sie diese Güter und Leistungen selbst besitzen beziehungsweise sich leisten könnten. Güter, die mehr als 80 oder 90 Prozent der Befragten für unbedingt erforderlich halten, sollten dann als Mindeststandards erklärt und zur Errechnung des erforderlichen Mindesteinkommens herangezogen werden.
Dieser Ansatz, so Hauser, könne am ehesten als unmittelbar demokratisch bezeichnet werden, weil hierbei alle Bürger als Experten gefragt seien. Dies wäre auch ein neuer Ansatzpunkt für die Bekämpfung der sozialen Ausgrenzung vieler Armer durch die übrige Bevölkerung. »Denn hierfür «, so Hauser, » ist die Sichtweise der nicht armen Bevölkerungsmehrheit im tägliche Umgang maßgeblich. «

Auch die von Armut betroffenen Menschen müssten beim Zustandekommen von nationalen Armutsberichten beteiligt werden forderte Jens Schröter von der Aktionsgemeinschaft arbeitsloser Bürgerinnen und Bürger in Bremen, der 1989 als Betroffener am Armutsbericht des Paritätischen mitgewirkt hat. Er war auch als Betroffenenvertreter in die Diskussionsrunde zum Abschluss der Tagung geladen. Dabei ging es unter anderem um die weitreichenden Folgen von Armut, Ausgrenzung und Benachteiligung, insbesondere aber die Folgen der Kinderarmut und die dringend notwendige bessere Unterstützung von Familien. An der Diskussion nahmen neben Schröter teil: Dr. Ulrich Schneider, Hauptgeschäftsführer des Paritätischen, Frauke Hunfeld, Journalistin und Autorin des Buches »Und plötzlich bist du arm «, Moderatorin Gwendolyn Stilling vom Paritätischen Gesamtverband, Dr. Armin Kuphal, Soziologe an der Uni des Saarlandes und ebenso wie Schneider Mitautor des ersten Armutsberichts des Paritätischen. 